

\section{INCORPORACIÓN DE HUELLA DE \\ CARBONO Y HUELLA ECOLÓGICA EN LAS BASES DE COSTES DE CONSTRUCCIÓN. ESTUDIO DE CASO DE UN PROYECTO DE URBANIZACIÓN EN ÉCIJA, ESPAÑA.}

\section{THE INCORPORATION OF CARBON FOOTPRINT AND ECOLOGICAL FOOTPRINT INTO CONSTRUCTION COST DATABASES: A CASE STUDY OF A DEVELOPMENT PROJECT IN ÉCIJA, SPAIN.}

ANTONIO FREIRE GUERRERO

Univerisdad de Sevilla, Departamento de Construcciones Arquitectónicas II

Sevilla, España

antoniowebmix@hotmail.com

JAVIER MUÑOZ MARTÍN

Univerisdad de Sevilla, Departamento de Construcciones Arquitectónicas $\|$

Sevilla, España

jmunoz@gmail.com
MADELYN MARRERO

Univerisdad de Sevilla, Departamento de Construcciones Arquitectónicas II

Sevilla, España

madelyn@us.es

\section{RESUMEN}

En el conjunto de factores que se consideran para diseñar, proyectar y tomar decisiones dentro de la construcción, se propone la inclusión de los indicadores "huella ecológica" y "huella de carbono" en las bases de costes de construcción (BCC), ya que constituyen herramientas simples y fáciles de utilizar que permiten integrar el aspecto ambiental en los presupuestos de los proyectos. Con el fin de demostrar dicha posibilidad, se toma como caso particular la BCC de Andalucía, para el cual se calcula un presupuesto ambiental. El modelo propuesto se valida con el análisis de un proyecto de urbanización industrial en Écija (España), proporcionando resultados comparables con otras metodologías de huella en edificación.

Palabras clave

Control de costes, huella ecológica, huella de carbono, urbanización

\section{ABSTRACT}

Among the set of factors taken into consideration during the design, planning and decision-making process of construction projects, the present article proposes that the ecological footprint and carbon footprint indicators be included in Construction Cost Databases (CCD), as they are simple, easy-to-use tools that make it possible to incorporate environmental aspects into project budgets. This research analyses the specific case of Andalusia's CCD in order to demonstrate the possibility of incorporating environmental aspects (ecological and carbon footprints) into the construction project budget, thereby creating a new "environmental budget". The proposed model is validated through the analysis of an industrial development project in Écija, Spain; the results obtained are comparable to those of other footprint methodologies in the construction sector.

Keywords 


\section{INTRODUCCIÓN.}

La construcción, y otros agentes intervinientes de forma directa o indirecta, tiene notables impactos ambientales en cuanto a consumo de recursos naturales y energía o emisión de gases de efecto invernadero, de ahí la necesidad de considerar la dimensión ambiental como clave en un enfoque de construcción sostenible. El proceso de la construcción es responsable de más del $40 \%$ de los recursos naturales, más de un $30 \%$ del consumo de energía y más de un $30 \%$ de las emisiones de gases de efecto invernadero (Cambio Global España, 2010). Además, es también responsable de una parte significativa del consumo de madera y de agua en el mundo.

La razón del impacto tan considerable de la construcción radica en los procesos de edificación, desde la fabricación de materiales, pasando por su construcción y posterior uso, hasta la demolición. Ante la necesidad de implantar mejoras en el aspecto ambiental, es necesario contar con indicadores contrastables y fiables que sean sensibles a los cambios. En esta línea, dos profesores de la Universidad de la Columbia Británica, Wackernagel y Rees, definieron el concepto de "huella ecológica" (HE) como "el área de territorio ecológicamente productivo (cultivos, pastos, bosques o ecosistemas acuático) necesaria para producir los recursos utilizados y para asimilar los residuos producidos, por una población dada, con un nivel de vida específico, de forma indefinida" (1996:. Mediante esta categoría es posible comparar la huella ambiental provocada por continentes, países, regiones, etc.

Entre los aspectos más relevantes de la HE se haya, en primer lugar, la simplicidad de sus conceptos, que además de ser fáciles de calcular, pueden ser entendidos y adoptados de manera general, con lo cual se facilita su influencia en las decisiones de consumo, legislación y regulación del sector; y, en segundo lugar, la unión de factores de diferentes fuentes en un solo indicador, que permite obtener una visión general de todos los impactos de una actividad o sector productivo. Esta unión hace posible estimar el impacto total de cualquier actividad humana de forma claramente comprensible, y ofrece también la opción de estudiar la HE por categoría (diferentes clasificaciones del territorio), para identificar de este modo sus aspectos más determinantes.

El indicador HE se ha utilizado desde su creación para la determinación de los impactos, en diferentes escalas: para predecir los impactos generados por la humanidad, para el cálculo periódico de la huella de la humanidad en el planeta o para calcular periódicamente la HE de diferentes países, ciudades, barrios, sectores productivos e industrias. Esta metodología, adaptada a las características únicas del sector de la construcción, ha sido elegida, en efecto, por su comprensibilidad, transparencia y capacidad de adaptación. En el área de la edificación, el indicador HE se ha aplicado al estudio del crecimiento de distritos de gran altura en Teherán (Samadpour y Faryadi, 2008), en viviendas de campesinos (Zhao y Mao, 2013), en hoteles (Li y Cheng, 2010) y en la rehabilitación de una casa centenaria (Bin y Parker, 2012); además de haberse desarrollado una herramienta para la estimación de la huella ecológica y huella de carbono de edificios (Olgyay, 2008). Asimismo, se ha analizado el ciclo de vida (Teng y Wu, 2014) de los edificios (proyecto, construcción, uso y demolición) y su estudio según la $\mathrm{HE}$ (energía, recursos, $\mathrm{CO}_{2}$ y residuos sólidos), aplicándolo a un centro de exposiciones en Wuhan (China). Cabe destacar otros dos estudios que han optado por adaptar el indicador HE a las particularidades del sector de la edificación (Bastianoni et al., 2007; Solís, 2011). Bastianoni calculó la HE de dos edificios italianos, teniendo en cuenta principalmente la energía incorporada en los materiales y en el proceso de construcción (un 5\% del total de los materiales). Los resultados de la HE se contabilizan en hectáreas de terreno necesario para la absorción del $\mathrm{CO}_{2}$, terreno de bosques (para los materiales de madera), superficie necesaria para la producción de los alimentos de los trabajadores y el área ocupada por los edificios.

Por otro lado, Solís, Marrero y Ramírez de Arellano (2013) han desarrollado un modelo de cálculo con algunas hipótesis innovadoras, tales como incluir el consumo de comida y los traslados de los operarios, o el consumo de agua en la obra, el cual no suele aparecer en los estudios de HE, ya que no está incluido en la metodología general del indicador. Con la inclusión de los alimentos, aparecen huellas adicionales asociadas a terrenos de cultivo, pastos y pesca, necesarios para su producción. El mismo modo de cálculo ha sido empleado con éxito para la estimación de la huella de carbono $(\mathrm{HC})$ (Solís, Martínez y Marrero, 2014; Solís et al., 2015) en el sector de la construcción.

Siguiendo estudios previos (Freire y Marrero, 2015a), donde se establecen las características de los diferentes sistemas de clasificación de la información de construcción (SCIC) y bases de costes de la construcción (BCC), se opta por desarrollar, como modelo de base de costes ambiental, la base de costes de construcción de Andalucía (BCCA), a la que serán incorporadas la HE y HC, permitiendo su aplicación en cualquier ámbito de la edificación (urbanización, rehabilitación, nueva construcción, etc.) y cualquier tipología de viviendas (plurifamiliares, unifamiliares aisladas o adosadas) (Figura 1).

\section{METODOLOGÍA.}

En la Figura 2 se representa el esquema general de la metodología a desarrollar, con los diferentes conceptos que forman parte de la HE clasificados en varios niveles (fuentes de impacto y huellas), procediendo a la explicación de cada uno de los elementos y las hipótesis adoptadas para su cálculo. El mismo esquema es válido para el cálculo de la HC, a excepción de que en este caso solo se evalúa la huella energética. 


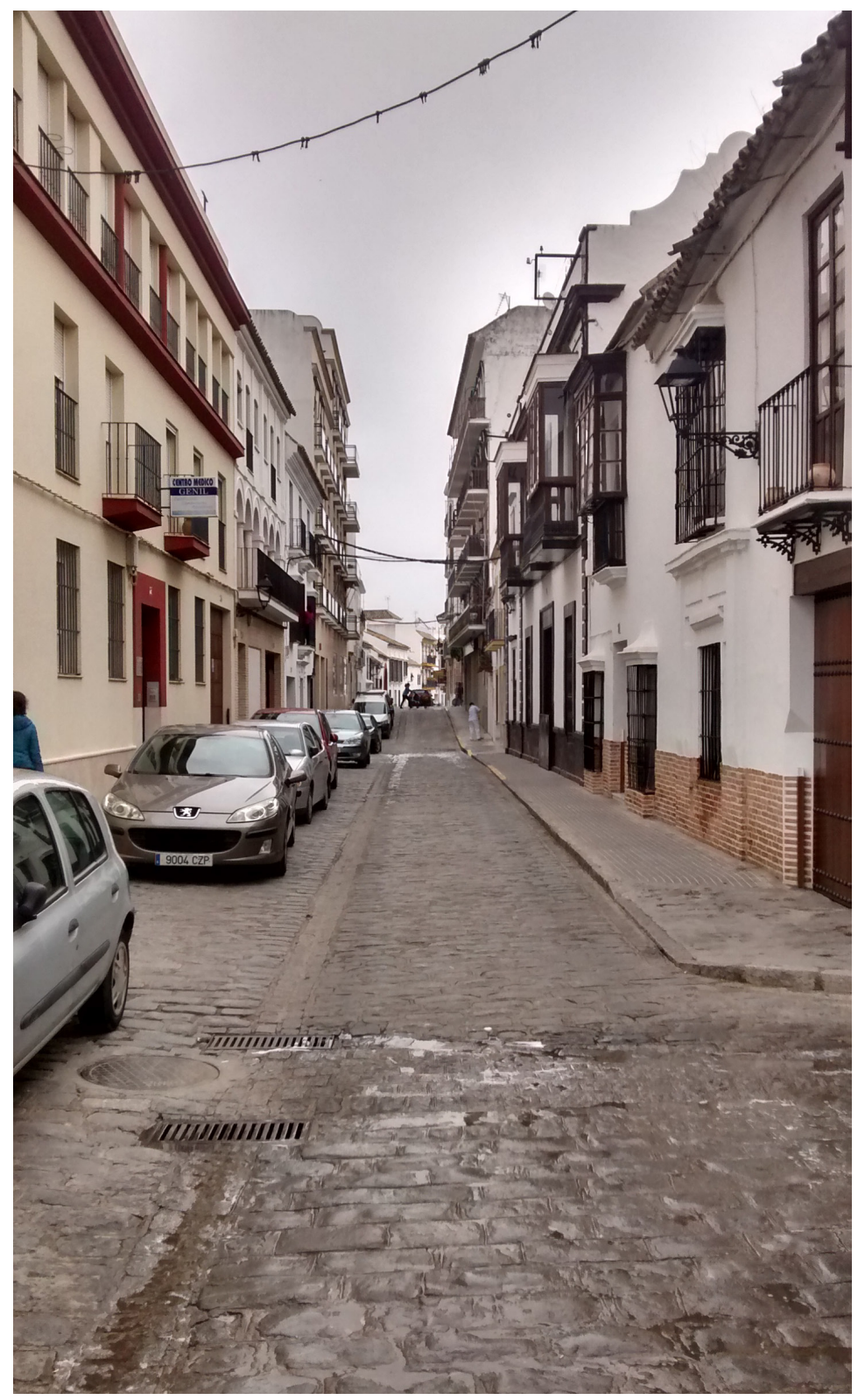

Figura 1. Viviendas adosadas en núcleo urbano. (Elaboración del autor, 2016)

\section{COSTES DIRECTOS.}

En este apartado se analizan los costes directos, que en los presupuestos tradicionales de construcción corresponden a los costes unitarios de maquinarias, mano de obra y materiales.

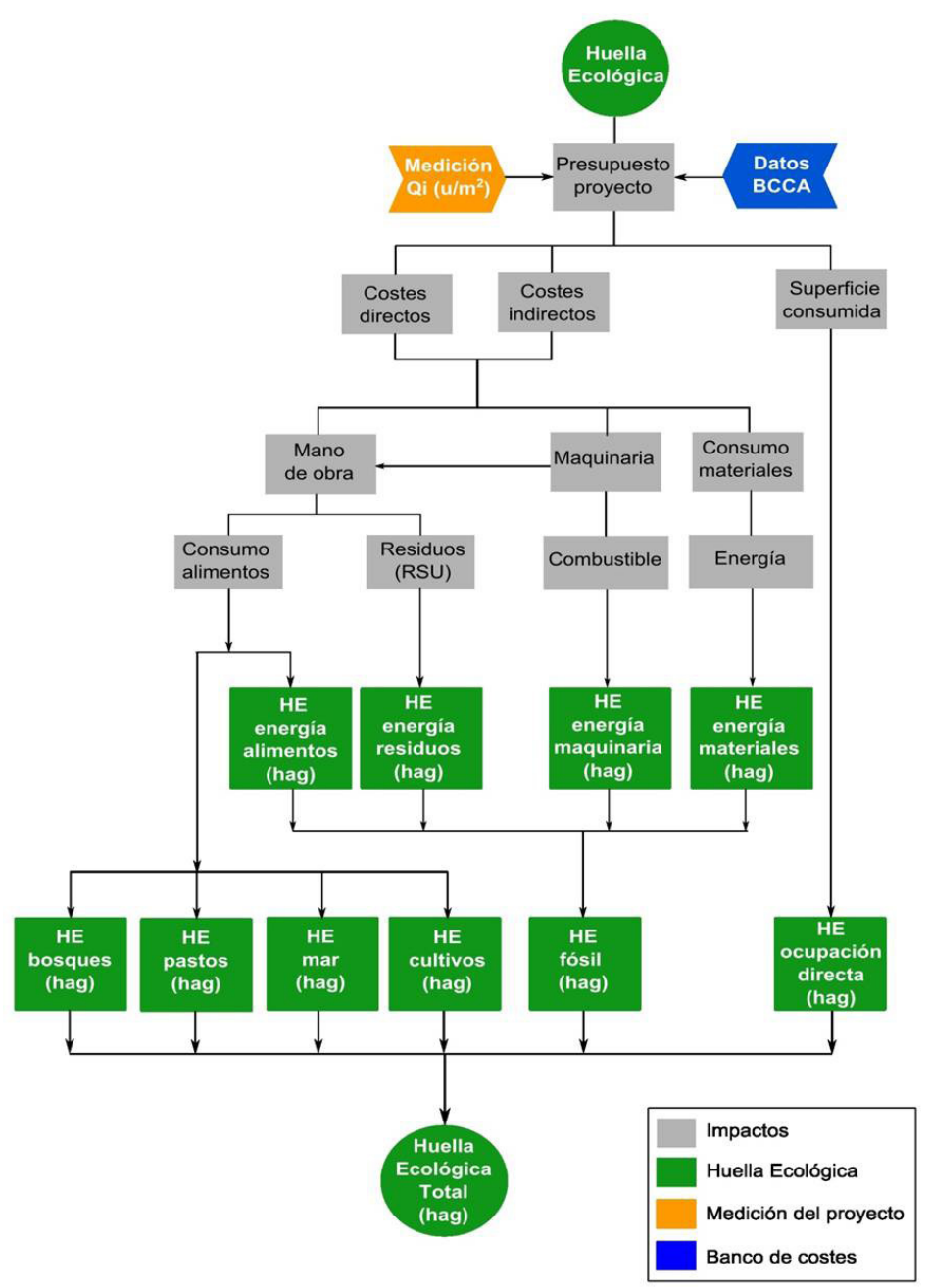

Figura 2. Metodología para el cálculo de la HE. (Elaboración del autor, 2016)

\section{MAQUINARIA.}

A partir de la metodología descrita por Freire y Marrero (2015b), adaptada para este caso, se analizan aquí las huellas provocadas por la utilización de maquinaria, concretamente por su consumo de energía (tanto consumo de combustible como energía eléctrica), vinculándola con la potencia de su motor. Cabe aclarar, que en este procedimiento se distingue la huella producida por el propio empleo de la maquinaria de la generada por el trabajador que la opera. 


\begin{tabular}{|c|c|c|c|c|c|c|c|}
\hline \multicolumn{8}{|c|}{ HE DE ALIMENTACIÓN EN ESPAÑA 2015} \\
\hline \multirow{2}{*}{\multicolumn{2}{|c|}{ Tipo Alimento }} & Cropland & $\begin{array}{l}\text { Grazing } \\
\text { Land }\end{array}$ & $\begin{array}{l}\text { Forest } \\
\text { Land }\end{array}$ & $\begin{array}{l}\text { Fishing } \\
\text { Grounds }\end{array}$ & $\begin{array}{l}\text { Carbon } \\
\text { Footprint }\end{array}$ & \multirow{2}{*}{ Total } \\
\hline & & Cultivos & Pastos & Bosques & Mar & Energía & \\
\hline \multicolumn{2}{|c|}{$\begin{array}{l}\text { HE (hag/persona y } \\
\text { año) }\end{array}$} & 0,57 & 0,13 & 0,01 & 0,33 & 0,10 & 1,15 \\
\hline \multicolumn{2}{|c|}{ Porcentaje } & $49,74 \%$ & $11,06 \%$ & $1,05 \%$ & $29,09 \%$ & $9,06 \%$ & $100,00 \%$ \\
\hline días/año & & 0,001564 & 0,000348 & 0,000033 & 0,000915 & 0,000285 & 0,003145 \\
\hline$\%$ almuerzo & $60 \%$ & 0,000939 & 0,000209 & 0,000020 & 0,000549 & 0,000171 & 0,001887 \\
\hline horas & 8,00 & 0,000117 & 0,000026 & 0,000002 & 0,000069 & 0,000021 & 0,000236 \\
\hline \multicolumn{2}{|c|}{$\begin{array}{c}\text { hag/hora trabajo y } \\
\text { almuerzo }\end{array}$} & 0,00012 & 0,00003 & 0,00000 & 0,00007 & 0,00002 & 0,00024 \\
\hline
\end{tabular}

Tabla 1. Datos para cálculo del coeficiente HEm. (Grunewald et al., 2015).

\section{MANO DE OBRA.}

En este aspecto, se analizan los impactos generados por los trabajadores: consumo de alimentos y la generación de residuos sólidos urbanos (RSU).

\section{HUELLAS PROVOCADAS POR ALIMENTOS.}

En la metodología descrita, las huellas producidas por la comida de los trabajadores se imputan a la huella de la construcción del edificio debido a que esta actividad se lleva a cabo en el lugar de trabajo, según plantea el modelo desarrollado por Doménech, donde las comidas de negocios se asignan a la HE corporativa. Para ello, es necesario obtener el número total de horas de mano de obra trabajadas en el proyecto de construcción. Las huellas se calculan usando la siguiente ecuación (Solís, 2011):

$H E_{\text {comida }}=H E_{m} \times h_{\text {totales }}$

Donde:

$\mathrm{HE}_{\text {comida }}$ : $\mathrm{HE}$ producida por el consumo de alimentos medida en hag/año.

$\mathrm{HE}_{\mathrm{m}}$ : HE de cada comida servida expresada en hag/hora de trabajador.

$\mathrm{h}_{\text {totales }}$ : total de horas trabajadas por todos los empleados.

Por lo tanto, es necesario obtener el coeficiente $\mathrm{HE}_{\mathrm{m}}$ del menú diario de cada trabajador, que genera cuatro tipos de territorios productivos: pastos, tierras de cultivo, mar productivo y bosques. Cada categoría de alimentos generará dos tipos de HE: la producida por el consumo del propio alimento (las carnes generarán $\mathrm{HE}$ de pastos, los pescados generarán $\mathrm{HE}$ de mar productivo, los cereales generarán $\mathrm{HE}$ de tierras de cultivo, etc.) y la que produce la manipulación y procesamiento de dicho alimento para ser ingerido, que será incluida en la HE fósil. Además, se tiene en cuenta la composición de un "menú tipo" en función del consumo de cada alimento con la productividad natural y el factor de equivalencia de cada territorio productivo (para huella de mar, pasto y cultivo), tal y como se muestran en la Tabla 1 (Grunewald et al., 2015).

\section{HE DE LOS RESIDUOS SÓLIDOS URBANOS (RSU).}

En la generación de residuos, se sigue un camino parecido al de la alimentación, ya que se utiliza un coeficiente que indica la generación media de RSU por trabajador (EUROSTAT, 2015) para obtener las toneladas totales de residuos producidos, frente al cálculo por naturaleza de los distintos RSU, obtenido de otros modelos ya publicados (González et al., 2015). A esta cifra, se le aplica las emisiones de $\mathrm{CO}_{2}$ por tonelada de residuo, la capacidad de absorción de emisiones que posee el territorio (IDAE, 2011) y el factor de equivalencia correspondiente al territorio productivo de bosques, como se muestra en la siguiente expresión:

$$
\left.H E_{R S U}=\left(H_{T R A B} \times R_{R S U} \times E_{R S U} \times 0,72\right) / A_{F}\right) \times F E_{B}
$$

Donde:

$\mathrm{H}_{\text {TRAB }}$ : horas trabajadas (h)

$\mathrm{R}_{\mathrm{RSU}}$ : cantidad de RSU producidos por hora de trabajo (toneladas/h $\mathrm{x}$ pers.)

$\mathrm{E}_{\mathrm{RSU}}$ : factor de emisión por residuo (toneladas $\mathrm{CO}_{2} / \mathrm{t} \mathrm{RSU}$ )

0,72: reducción de emisiones a absorber (28\%)

$\mathrm{A}_{\mathrm{E}}$ : productividad del área de absorción de carbono, o factor de absorción ( $\mathrm{tCO}_{2} / \mathrm{ha}$ )

$\mathrm{FE}_{\mathrm{B}}$ : factor de equivalencia de los bosques (hag/ha)

\section{MATERIALES.}

Para calcular la huella producida por los materiales, se tiene en cuenta la energía que se necesita durante el ciclo de vida de la cuna a la puerta. También se realiza un análisis del transporte del material, estableciendo una hipótesis de distancia recorrida por los medios de transporte y siguiendo, luego, el procedimiento indicado para las maquinarias, con el fin de convertir este dato en HE. Este factor introduce como principales novedades el análisis e inclusión del transporte de los materiales de construcción desde la fábrica hasta la obra, y la obtención de diversas "familias ambientales" procedentes de bases de datos de análisis de ciclo de vida de materiales (ACV), lo que permite obtener las emisiones de cada material producidas durante su fabricación. 


\begin{tabular}{|c|c|c|c|c|c|}
\hline UD & MATERIAL & $\begin{array}{l}\text { EMISIONES } \\
(\mathrm{kgCO} / \mathrm{kg})\end{array}$ & UD & MATERIAL & $\begin{array}{l}\text { EMISIONES } \\
(\mathrm{kgCO} / \mathrm{kg})\end{array}$ \\
\hline \multicolumn{3}{|c|}{ AISLANTES } & \multicolumn{3}{|c|}{ MORTEROS } \\
\hline $\mathrm{kg}$ & $\begin{array}{l}\text { Elastómero aislante de } \\
\text { tubos }\end{array}$ & 4,060 & $\mathrm{~kg}$ & Cal apagada suelta & 0,739 \\
\hline $\mathrm{kg}$ & $\begin{array}{l}\text { Espuma de urea } \\
\text { formaldehido in situ }\end{array}$ & 2,897 & $\mathrm{~kg}$ & $\begin{array}{l}\text { Cal apagada } \\
\text { empaquetada }\end{array}$ & 0,749 \\
\hline $\mathrm{kg}$ & Espuma de vidrio & 1,500 & $\mathrm{~kg}$ & Cal hidráulica & 0,805 \\
\hline $\mathrm{kg}$ & $\begin{array}{l}\text { Fibra de celulosa (incluido } \\
\text { soplado) }\left(d=50 \mathrm{~kg} / \mathrm{m}^{3}\right)\end{array}$ & 0,260 & $\mathrm{~kg}$ & Cal viva en cascotes suelta & 0,960 \\
\hline $\mathrm{kg}$ & Fibra de vidrio & 2,408 & $\mathrm{~kg}$ & Cal viva suelta & 0,962 \\
\hline $\mathrm{kg}$ & $\begin{array}{l}\text { Lámina de poliestireno } \\
\text { expandido }\end{array}$ & 3,355 & $\mathrm{~kg}$ & Cal viva empaquetada & 0,971 \\
\hline $\mathrm{kg}$ & $\begin{array}{l}\text { Lámina de urea } \\
\text { formaldehido rígida }\end{array}$ & 2,776 & $\mathrm{~kg}$ & Cemento (genérico) & 0,758 \\
\hline $\mathrm{kg}$ & Lana de roca & 1,368 & $\mathrm{~kg}$ & $\begin{array}{l}\text { Cemento con escoria de } \\
\text { alto horno }\end{array}$ & 0,442 \\
\hline $\mathrm{kg}$ & Lana de roca, empaquetada & 1,262 & $\mathrm{~kg}$ & $\begin{array}{l}\text { Cemento Portland con } \\
\text { escoria de alto horno }\end{array}$ & 0,701 \\
\hline $\mathrm{kg}$ & Lana de vidrio en rollo & 1,387 & $\mathrm{~kg}$ & $\begin{array}{l}\text { Cemento Portland } \\
\text { calcáreo }\end{array}$ & 0,715 \\
\hline $\mathrm{kg}$ & Poliestireno extruido (XPS) & 2,901 & $\mathrm{~kg}$ & $\begin{array}{l}\text { Cemento Portland II/A-L } \\
42,5 \mathrm{R}\end{array}$ & 0,818 \\
\hline $\mathrm{kg}$ & Poliuretano (espuma) & 3,498 & $\mathrm{~kg}$ & Cemento Portland 52,5 R & 0,828 \\
\hline \multicolumn{3}{|c|}{ ÁRIDOS Y PIEDRAS } & $\mathrm{kg}$ & Estuco & 0,069 \\
\hline $\mathrm{kg}$ & Arcilla & 0,003 & $\mathrm{~kg}$ & Mortero de cal & 0,596 \\
\hline $\mathrm{kg}$ & Arcilla expandida & 0,349 & $\mathrm{~kg}$ & Mortero de cemento & 0,191 \\
\hline $\mathrm{kg}$ & Arena & 0,002 & $\mathrm{~kg}$ & Mortero ligero & 0,471 \\
\hline $\mathrm{kg}$ & Arena de Sílice & 0,021 & $\mathrm{~kg}$ & Pasta adhesiva & 1,023 \\
\hline $\mathrm{kg}$ & Grava genérica & 0,003 & $\mathrm{~kg}$ & Pasta de arcilla & 0,018 \\
\hline $\mathrm{kg}$ & Grava en bolos & 0,002 & $\mathrm{~kg}$ & $\begin{array}{l}\text { Suelo continuo de } \\
\text { cemento }\end{array}$ & 0,171 \\
\hline $\mathrm{kg}$ & Grava de machaqueo & 0,004 & $\mathrm{~kg}$ & Tabla de fibra de yeso & 0,279 \\
\hline $\mathrm{kg}$ & Bloque de arenisca & 0,060 & $\mathrm{~kg}$ & Tabla de yeso laminado & 0,335 \\
\hline $\mathrm{kg}$ & Perlita & 0,001 & $\mathrm{~kg}$ & Yeso (mineral) & 0,002 \\
\hline $\mathrm{kg}$ & Perlita expandida & 0,952 & \multicolumn{3}{|c|}{ PINTURAS } \\
\hline $\mathrm{kg}$ & Piedra caliza & 0,002 & $\mathrm{~kg}$ & Aglutinante acrílico & 1,279 \\
\hline $\mathrm{kg}$ & $\begin{array}{l}\text { Piedra caliza machacada } \\
\text { Dara moler }\end{array}$ & 0,002 & $\mathrm{~kg}$ & Barniz acrílico & 1,738 \\
\hline
\end{tabular}

Tabla 2. Familias medioambientales de las materias primas. Parte 1. (Elaboración del autor, 2016)

En el caso de los materiales, las emisiones producidas durante la fabricación de las materias primas en productos de construcción, serán contabilizadas en hectáreas, a diferencia de otras metodologías en las que se calculaba la energía y se le aplicaba un factor de emisiones medio (González, Marrero y Solís, 2015).

El primer paso a realizar para poder obtener la HE de cada elemento consiste en efectuar una conversión de la unidad de medida de origen de cada precio básico (metros, metros cuadrados, metros cúbicos, toneladas, millares) a metros cúbicos, de modo que se pueda emplear la densidad establecida en los documentos de apoyo utilizados (Catálogo de Soluciones Constructivas del Código Técnico de la Edificación (CSC) y el Documento Básico de Seguridad Estructural del Código Técnico de la Edificación. Acciones en la Edificación (DB-SE AE)), para obtener el peso de cada elemento (Freire y Marrero, 2015a). Una vez obtenido dicho peso, se acudirá a las bases de datos de análisis de ciclo de vida (ACV), las cuales definen las emisiones que contiene cada kilogramo de material, con lo que se obtiene la HC.

Entre las diferentes bases de datos de $A C V$, se opta por Ecoinvent, implementada en Symapro y desarrollada por el Swiss Center for Life Cycle Inventories, debido a su transparencia en el desarrollo de procesos (informes, diagramas de flujo, metodología), consistencia, referencias y el destacado hecho de que fusiona información de varias bases de datos de la industria de la construcción (Martínez, 2016). De este repositorio, se han obtenido "familias medioambientales" que serán las encargadas de asignar a cada precio básico sus correspondientes emisiones en función de su similitud. En las Tablas 2 y 3 se exponen los materiales básicos y sus procesos o transformaciones (formación de tubos, empaquetado de áridos, formación de piezas de metal). 


\begin{tabular}{|c|c|c|c|c|c|}
\hline UD & MATERIAL & $\begin{array}{l}\text { EMISIONES } \\
\left(\mathrm{kgCO}_{2} / \mathrm{kg}\right)\end{array}$ & UD & MATERIAL & $\begin{array}{l}\text { EMISIONES } \\
\left(\mathrm{kgCO}_{2} / \mathrm{kg}\right)\end{array}$ \\
\hline \multicolumn{3}{|c|}{ ÁRIDOS Y PIEDRAS } & \multicolumn{3}{|c|}{ PINTURAS } \\
\hline $\mathrm{kg}$ & $\begin{array}{l}\text { Piedra caliza machacada } \\
\text { lavada }\end{array}$ & 0,002 & $\mathrm{~kg}$ & Betún & 0,382 \\
\hline $\mathrm{kg}$ & Piedra caliza molida suelta & 0,012 & $\mathrm{~kg}$ & $\begin{array}{l}\text { Lámina de betún modificado, } \\
\text { unión en caliente }\end{array}$ & 0,486 \\
\hline $\mathrm{kg}$ & $\begin{array}{l}\text { Piedra caliza molida } \\
\text { empaquetada }\end{array}$ & 0,022 & $\mathrm{~kg}$ & $\begin{array}{l}\text { Lámina de betún modificado, } \\
\text { unión en frío }\end{array}$ & 0,353 \\
\hline $\mathrm{kg}$ & $\begin{array}{l}\text { Piedra natural, placa para } \\
\text { suelo }\end{array}$ & 0,342 & $\mathrm{~kg}$ & Pintura (disolvente: agua) & 2,212 \\
\hline $\mathrm{kg}$ & Piedra natural, placa cortada & 0,251 & $\mathrm{~kg}$ & Pintura (disolvente: otro) & 2,212 \\
\hline $\mathrm{kg}$ & Piedra natural, placa pulida & 0,415 & $\mathrm{~kg}$ & $\begin{array}{l}\text { Sellante bituminoso o slurry } \\
\text { asfáltico }\end{array}$ & 0,970 \\
\hline \multicolumn{3}{|c|}{ CERÁMICOS } & \multicolumn{3}{|c|}{ PLÁSTICOS } \\
\hline $\mathrm{kg}$ & Baldosa cerámica & 0,766 & $\mathrm{~kg}$ & ABS (plástico) & 3,121 \\
\hline $\mathrm{kg}$ & $\begin{array}{l}\text { Cerámica sanitarios } \\
\text { (porcelana) }\end{array}$ & 2,258 & $\mathrm{~kg}$ & $\begin{array}{l}\text { Carpintería de ventana de } \\
\text { PVC (esp } 10 \mathrm{~cm} \text { y } d=1.390 \mathrm{~kg} / \\
\mathrm{m}^{3} \text { ) }\end{array}$ & 1,693 \\
\hline $\mathrm{kg}$ & $\begin{array}{l}\text { Fibrocemento (chapa } \\
\text { ondulada) }\end{array}$ & 0,623 & $\mathrm{~kg}$ & $\begin{array}{l}\text { Etileno-acetato de vinilo } \\
\text { (caucho-vinilo) }\end{array}$ & 1,815 \\
\hline $\mathrm{kg}$ & Fibrocemento (baldosa) & 0,783 & $\mathrm{~kg}$ & Fenólico (resina) & 3,702 \\
\hline $\mathrm{kg}$ & Fibrocemento (tejas) & 0,691 & $\mathrm{~kg}$ & Goma sintética & 2,394 \\
\hline $\mathrm{kg}$ & Ladrillo o bloque cerámico & 0,212 & $\mathrm{~kg}$ & Látex & 2,422 \\
\hline $\mathrm{kg}$ & $\begin{array}{l}\text { Ladrillo refractario básico } \\
\text { empaquetado }\end{array}$ & 2,233 & $\mathrm{~kg}$ & Metacrilato & 5,566 \\
\hline $\mathrm{kg}$ & $\begin{array}{l}\text { Ladrillo refractario de arcilla } \\
\text { empaquetado }\end{array}$ & 1,123 & $\mathrm{~kg}$ & $\begin{array}{l}\text { Poliamida reforzada con fibra } \\
\text { de vidrio }\end{array}$ & 7,629 \\
\hline $\mathrm{kg}$ & $\begin{array}{l}\text { Ladrillo refractario alto en } \\
\text { óxido alum., empaquetado }\end{array}$ & 0,851 & $\mathrm{~kg}$ & Poliéster (resina) & 4,268 \\
\hline \multicolumn{3}{|c|}{ HORMIGONES } & $\mathrm{kg}$ & $\begin{array}{l}\text { Poliéster reforzado con fibra } \\
\text { de vidrio }\end{array}$ & 3,378 \\
\hline $\mathrm{kg}$ & Baldosa de hormigón & 0,225 & $\mathrm{~kg}$ & Poliestireno GPPS (general) & 2,754 \\
\hline $\mathrm{kg}$ & Bloque de hormigón & 0,121 & $\mathrm{~kg}$ & $\begin{array}{l}\text { Poliestireno expandible (para } \\
\text { EPS) }\end{array}$ & 2,594 \\
\hline $\mathrm{kg}$ & $\begin{array}{l}\text { Bloque de hormigón aireado } \\
\text { en autoclave }\end{array}$ & 0,403 & $\mathrm{~kg}$ & $\begin{array}{l}\text { Poliestireno de alto impacto } \\
\text { (HIPS) }\end{array}$ & 2,794 \\
\hline $\mathrm{kg}$ & Teja de hormigón & 0,206 & $\mathrm{~kg}$ & $\begin{array}{l}\text { Polietileno de alta densidad } \\
\text { (HDPE) }\end{array}$ & 1,573 \\
\hline $\mathrm{kg}$ & Hormigón $\left(\mathrm{d}=2380 \mathrm{~kg} / \mathrm{m}^{3}\right)$ & 0,110 & $\mathrm{~kg}$ & $\begin{array}{l}\text { Polietileno de baja densidad } \\
\text { (LDPE) }\end{array}$ & 1,694 \\
\hline $\mathrm{kg}$ & $\begin{array}{l}\text { Hormigón de cimentación } \\
\left(\mathrm{d}=2385 \mathrm{~kg} / \mathrm{m}^{3}\right)\end{array}$ & 0,067 & $\mathrm{~kg}$ & $\begin{array}{l}\text { Polietileno de baja densidad } \\
\text { linear (LLDPE) }\end{array}$ & 1,495 \\
\hline $\mathrm{kg}$ & $\begin{array}{l}\text { Hormigón pobre (limpieza) } \\
\left(\mathrm{d}=2190 \mathrm{~kg} / \mathrm{m}^{3}\right)\end{array}$ & 0,056 & $\mathrm{~kg}$ & Polietileno tereftalato (PET) & 2,421 \\
\hline $\mathrm{kg}$ & $\begin{array}{l}\text { Hormigón de alta resistencia } \\
\left(\mathrm{d}=2440 \mathrm{~kg} / \mathrm{m}^{3}\right)\end{array}$ & 0,133 & $\mathrm{~kg}$ & Polipropileno & 1,679 \\
\hline $\mathrm{kg}$ & $\begin{array}{l}\text { Hormigón de alta resistencia } \\
\text { contra heladas }(\mathrm{d}=2440 \mathrm{~kg} / \\
\left.\mathrm{m}^{3}\right)\end{array}$ & 0,118 & $\mathrm{~kg}$ & $\begin{array}{l}\text { Poliuretano (espuma flexible, } \\
\text { camas y muebles) }\end{array}$ & 4,028 \\
\hline \multicolumn{3}{|c|}{ MADERAS } & $\mathrm{kg}$ & PVC & 1,835 \\
\hline $\mathrm{kg}$ & $\begin{array}{l}\text { Carpintería de ventana } \\
\text { madera (esp } 10 \mathrm{~cm} \text { y } \mathrm{d}= \\
5 \mathrm{KN} / \mathrm{m}^{3} \text { ) }\end{array}$ & 1,026 & $\mathrm{~kg}$ & Resina epoxi & 5,940 \\
\hline $\mathrm{kg}$ & Corcho (baldosa o lámina) & $-0,739$ & $\mathrm{~kg}$ & $\begin{array}{l}\text { Sellante de goma natural } \\
\text { (caucho) }\end{array}$ & 1,690 \\
\hline $\mathrm{kg}$ & Lana de madera & $-1,600$ & $\mathrm{~kg}$ & Sellante de polisulfido & 1,258 \\
\hline $\mathrm{kg}$ & $\begin{array}{l}\text { Madera blanda (resto) } \\
\left(=600 \mathrm{~kg} / \mathrm{m}^{3}\right)\end{array}$ & $-1,227$ & $\mathrm{~kg}$ & $\begin{array}{l}\text { Tetrafluoroetileno (sustitutivo } \\
\text { vidrios exteriores) }\end{array}$ & 9,805 \\
\hline $\mathrm{kg}$ & $\begin{array}{l}\text { Mad. dura (ébano, boj, } \\
\text { encina) }\left(\mathrm{d}=1.100 \mathrm{~kg} / \mathrm{m}^{3}\right)\end{array}$ & $-0,998$ & \multicolumn{3}{|c|}{ TEXTILES } \\
\hline $\mathrm{kg}$ & Papel & $-0,675$ & $\mathrm{~kg}$ & Algodón (fibra desmotada) & $-0,041$ \\
\hline $\mathrm{kg}$ & Papel reciclado & $-0,648$ & $\mathrm{~kg}$ & Algodón tejido & 19,512 \\
\hline $\mathrm{kg}$ & $\begin{array}{l}\text { Puerta interior madera ciega } \\
\text { (esp } 10 \mathrm{~cm} \text { y } d=5 \mathrm{KN} / \mathrm{m}^{3} \text { ) }\end{array}$ & $-0,802$ & $\mathrm{~kg}$ & Algodón en hilo & 8,909 \\
\hline
\end{tabular}




\begin{tabular}{|c|c|c|c|c|c|}
\hline UD & MATERIAL & $\begin{array}{l}\text { EMISIONES } \\
(\mathrm{kgCO} / \mathrm{kg})\end{array}$ & UD & MATERIAL & $\begin{array}{l}\text { EMISIONES } \\
(\mathrm{kgCO} / \mathrm{kg})\end{array}$ \\
\hline \multicolumn{3}{|c|}{ MADERAS } & \multicolumn{3}{|c|}{ TEXTILES } \\
\hline \multirow{2}{*}{$\begin{array}{l}\mathrm{kg} \\
\mathrm{kg}\end{array}$} & \multirow{3}{*}{$\begin{array}{l}\text { Puerta interior madera-vidrio } \\
\text { (esp } 10 \mathrm{~cm} \text { y } \mathrm{d}=5 \mathrm{Kn} / \mathrm{m}^{3} \text { ) } \\
\text { Puerta exterior madera- } \\
\text { vidrio (esp } 10 \mathrm{~cm} \text { y } \mathrm{d}=5 \mathrm{kN} / \\
\left.\mathrm{m}^{3}\right) \\
\text { Tablero de lana de madera } \\
\text { aglomerada con cemento } \\
\left(\mathrm{d}=5 \mathrm{kN} / \mathrm{m}^{3}\right)\end{array}$} & $-0,323$ & $\mathrm{~kg}$ & Lana & 32,600 \\
\hline & & 1,077 & \multicolumn{3}{|c|}{ VARIOS } \\
\hline kg & & $-0,097$ & $\mathrm{~kg}$ & $\begin{array}{l}\text { Agua potable en usuario } \\
\text { final }\end{array}$ & 0,000 \\
\hline \multicolumn{3}{|c|}{ METALES } & $\mathrm{kg}$ & Emulsión asfáltica & 0,193 \\
\hline $\mathrm{kg}$ & $\begin{array}{l}\text { Acero de baja aleación } \\
\text { (genérico) }\end{array}$ & 1,623 & $\mathrm{~kg}$ & Explosivo & 1,384 \\
\hline $\mathrm{kg}$ & Acero cromado (inoxidable) & 4,906 & $\mathrm{~kg}$ & $\begin{array}{l}\text { Tensioactivos (prod. } \\
\text { limpieza) }\end{array}$ & 1,872 \\
\hline $\mathrm{kg}$ & Acero reforzado & 1,344 & $\mathrm{~kg}$ & Plastificante & 4,405 \\
\hline $\mathrm{kg}$ & Aluminio mixto (genérico) & 6,698 & \multicolumn{3}{|c|}{ VIDRIOS } \\
\hline $\mathrm{kg}$ & Aluminio primario & 9,533 & $\mathrm{~kg}$ & Vidrio plano no recubierto & 0,520 \\
\hline kg & Bronce & 2,682 & \multirow{8}{*}{$\mathrm{kg}$} & \multirow[t]{8}{*}{ Vidrio plano recubierto } & \multirow[t]{8}{*}{0,628} \\
\hline $\mathrm{kg}$ & Cobre (genérico) & 1,853 & & & \\
\hline $\mathrm{kg}$ & Cobre primario & 1,785 & & & \\
\hline $\mathrm{kg}$ & Hierro de fundición & 1,343 & & & \\
\hline $\mathrm{kg}$ & Latón & 2,364 & & & \\
\hline $\mathrm{kg}$ & $\begin{array}{l}\text { Pellets de hierro, de la } \\
\text { fabricación }\end{array}$ & 0,079 & & & \\
\hline $\mathrm{kg}$ & Plomo & 0,998 & & & \\
\hline $\mathrm{kg}$ & Zinc & 3,165 & & & \\
\hline
\end{tabular}

\begin{tabular}{|c|c|c|c|c|c|}
\hline UD & MATERIAL & $\begin{array}{l}\text { EMISIONES } \\
(\mathbf{k g C O} / \mathbf{k g})\end{array}$ & UD & MATERIAL & $\begin{array}{l}\text { EMISIONES } \\
(\mathrm{kgCO}, / \mathrm{kg})\end{array}$ \\
\hline \multicolumn{3}{|c|}{ ÁRIDOS Y PIEDRAS } & \multicolumn{3}{|c|}{ MORTEROS } \\
\hline kg & Arcilla en saco & 0,008 & kg & Cal hidráulica en saco & 0,814 \\
\hline $\mathrm{kg}$ & Arcilla expandida & 0,349 & $\mathrm{~kg}$ & Cal viva en cascotes en saco & 0,960 \\
\hline $\mathrm{kg}$ & Arcilla expandida en saco & 0,354 & $\mathrm{~kg}$ & Cemento (genérico) en saco & 0,009 \\
\hline $\mathrm{kg}$ & Arena & 0,002 & $\mathrm{~kg}$ & Cemento cola & 0,969 \\
\hline $\mathrm{kg}$ & Grava de machaqueo & 0,004 & $\mathrm{~kg}$ & $\begin{array}{l}\text { Cemento con escoria de alto } \\
\text { horno en saco } \\
\text { Cemento Portland con }\end{array}$ & 0,758 \\
\hline $\mathrm{kg}$ & Grava en bolos & 0,002 & $\mathrm{~kg}$ & $\begin{array}{l}\text { escoria de alto horno en } \\
\text { saco }\end{array}$ & 0,005 \\
\hline $\mathrm{kg}$ & $\begin{array}{l}\text { Caliza machacada (albero, } \\
\text { marmolina, polvo mármol) }\end{array}$ & 0,002 & $\mathrm{~kg}$ & $\begin{array}{l}\text { Cemento Portland calcáreo } \\
\text { en saco }\end{array}$ & 0,763 \\
\hline $\mathrm{kg}$ & Arena de Sílice & 0,021 & $\mathrm{~kg}$ & $\begin{array}{l}\text { Cemento Portland } 42,5 \mathrm{R} \text { en } \\
\text { saco }\end{array}$ & 0,758 \\
\hline $\mathrm{kg}$ & Arena de sílice empaquetada & 0,026 & $\mathrm{~kg}$ & $\begin{array}{l}\text { Cemento Portland 52,5 R en } \\
\text { saco }\end{array}$ & 1,279 \\
\hline \multicolumn{3}{|c|}{ CERÁMICOS } & $\mathrm{kg}$ & Mortero de cemento & 1,520 \\
\hline $\mathrm{kg}$ & Ladrillo o bloque cerámico & 0,212 & $\mathrm{~kg}$ & $\begin{array}{l}\text { Tabla de yeso laminado y } \\
\text { fibra de vidrio }\end{array}$ & 0,442 \\
\hline
\end{tabular}




\begin{tabular}{|c|c|c|c|c|c|}
\hline UD & MATERIAL & $\begin{array}{l}\text { EMISIONES } \\
\left(\mathrm{kgCO}_{2} / \mathrm{kg}\right)\end{array}$ & UD & MATERIAL & $\begin{array}{l}\text { EMISIONES } \\
\left(\mathrm{kgCO}_{2} / \mathrm{kg}\right)\end{array}$ \\
\hline \multicolumn{3}{|c|}{ HORMIGONES } & \multicolumn{3}{|c|}{ MORTEROS } \\
\hline $\mathrm{kg}$ & $\begin{array}{l}\text { Bloque de hormigón aireado en } \\
\text { autoclave }\end{array}$ & 0,403 & $\mathrm{~kg}$ & \multirow{2}{*}{$\begin{array}{l}\text { Tabla de yeso laminado y } \\
\text { poliestireno expandido } \\
\text { Tabla de celulosa de } \\
\text { cemento }\end{array}$} & 95,550 \\
\hline $\mathrm{kg}$ & Hormigón (d=2380kg/m3) & 0,110 & $\mathrm{~kg}$ & & 0,446 \\
\hline $\mathrm{kg}$ & Bloque de hormigón & 0,121 & \multicolumn{3}{|c|}{ PLASTICOS } \\
\hline \multicolumn{3}{|c|}{ MADERAS } & $\mathrm{kg}$ & Película de PVC & 71,200 \\
\hline m3 & Madera blanda tratada & $-730,309$ & $\mathrm{~kg}$ & Tubo de polietileno & 0,402 \\
\hline m3 & Madera dura tratada & $-1091,709$ & $\mathrm{~kg}$ & Tubo de polipropileno & 2,237 \\
\hline $\mathrm{kg}$ & Papel reciclado & $-0,648$ & $\mathrm{~kg}$ & Tubo de PVC & 1,573 \\
\hline m3 & $\begin{array}{l}\text { Tablero de lana de madera } \\
\text { aglomerada con cemento }\end{array}$ & $-49,399$ & $\mathrm{~kg}$ & Pieza PVC & 0,312 \\
\hline \multicolumn{3}{|c|}{ METALES } & $\mathrm{kg}$ & Bloque de poliestireno & 116,700 \\
\hline $\mathrm{kg}$ & Cable de aluminio & 8,760 & $\mathrm{~kg}$ & $\begin{array}{l}\text { Pieza polietileno (enchufe, } \\
\text { interruptor...) }\end{array}$ & 1,679 \\
\hline $\mathrm{kg}$ & Cable de cobre & 1,853 & $\mathrm{~kg}$ & Pieza poliéster & 0,312 \\
\hline $\mathrm{m} 2$ & Carpintería de aluminio anodizado & 2,062 & $\mathrm{~kg}$ & Pieza ABS & 1,991 \\
\hline $\mathrm{m} 2$ & Carpintería de aluminio lacado & 3,915 & $\mathrm{~kg}$ & Pieza Polipropileno & 1,835 \\
\hline $\mathrm{kg}$ & Lámina Aluminio & 397,058 & $\mathrm{~kg}$ & Pieza/Plancha Poliuretano & 0,312 \\
\hline $\mathrm{kg}$ & Lámina de acero & 3,841 & $\mathrm{~kg}$ & Tubería polietileno & 2,147 \\
\hline $\mathrm{kg}$ & Perfil de acero laminado & 400,899 & $\mathrm{~kg}$ & $\begin{array}{l}\text { Tubería etileno-acetato } \\
\text { vinilo (caucho-vinilo) }\end{array}$ & 1,835 \\
\hline $\mathrm{kg}$ & Pieza de acero cromado/galvanizado & 397,058 & $\mathrm{~kg}$ & Plancha poliestireno & 1,179 \\
\hline $\mathrm{kg}$ & Pieza de aluminio & 8,188 & $\mathrm{~kg}$ & Película de polietileno & 2,096 \\
\hline $\mathrm{kg}$ & Tubería de cobre & 2,202 & & & \\
\hline $\mathrm{kg}$ & Tubería de acero & 1,623 & & & \\
\hline $\mathrm{kg}$ & Pieza latón & 0,349 & & & \\
\hline $\mathrm{kg}$ & Pieza bronce & 1,972 & & & \\
\hline
\end{tabular}

Tabla 3. Familias medioambientales de los materiales con transformaciones. Parte 2. (Elaboración del autor, 2016)

\section{COSTES INDIRECTOS.}

Siguiendo el enfoque de Freire y Marrero (2015a) para el cálculo de la energía incorporada en los costes indirectos (elementos que no pueden imputarse a una actividad única dentro de la obra), se efectúa a continuación el análisis de la HE. Para este efecto, se debe realizar un estudio de los costes indirectos atribuibles a cualquier proyecto de edificación, tomando como referencia la BCCA y recogiendo todos los elementos que conforman este grupo. Cada concepto se traduce en datos útiles para calcular la HE (horas anuales efectivas de trabajadores, consumos de combustible por la maquinaria, consumo de agua y electricidad en las casetas de obra, etc.), empleando la metodología descrita en las secciones previas, las diferentes conversiones y aplicación de coeficientes para obtener la HE de la mano de obra, maquinaria, consumo eléctrico y de agua.

\section{SUPERFICIE CONSUMIDA.}

Para la estimación de la HE de la superficie consumida se tiene en cuenta el suelo que se ocupa directamente, ya que será biológicamente improductivo desde el momento en que sea urbanizado. En el presente análisis dicha superficie responderá a la que se considerará en el proyecto.

Por tanto, la HE de superficie ocupada, vendrá dada por la transformación sufrida por el suelo a analizar. Dicha huella se obtendrá calculando la superficie a través de la memoria de cada proyecto analizado. No se aplica ningún factor de conversión, ya que se trata de unidades de superficie (ha), pero sí se aplica el factor de equivalencia correspondiente al territorio donde se actúa; tal y como se muestra en la siguiente expresión (Solís, 2011):

$\mathrm{HE}_{\text {sup }}=\mathrm{S} \times \mathrm{FE}_{\mathrm{x}}$

Donde:

S: superficie de ocupación directa en hectáreas (ha).

$\mathrm{FE}_{\mathrm{x}}$ : factor de equivalencia de la superficie construida (hag/ ha). En el indicador se definen dos posibles tipos de territorio: bosques o cultivos. 


\begin{tabular}{|l|c|c|c|c|c|c|}
\hline \multicolumn{1}{|c|}{ Huella Total } \\
\hline Impacto & Fósil & Pastos & Bosques & Mar & Cultivos & $\begin{array}{c}\text { Superficie } \\
\text { construida }\end{array}$ \\
\hline Maquinaria & $12.937,81$ & 11,89 & 7,35 & 31,27 & 52,72 & \\
\hline Mano de obra & 8,75 & 5,02 & 0,47 & 13,20 & 52,72 & \\
\hline Costes indirectos & 211,12 & 2,22 & 0,21 & 5,84 & 9,98 & 0,21 \\
\hline Materiales & $6.576,30$ & & & & & \\
\hline Total & $\mathbf{1 9 . 7 3 3 , 9 9}$ & $\mathbf{1 9 , 1 3}$ & $\mathbf{8 , 0 3}$ & $\mathbf{5 0 , 3 0}$ & $\mathbf{1 1 5 , 4 1}$ & $\mathbf{0 , 2 1}$ \\
\hline
\end{tabular}

Tabla 4. Resultados de caso práctico. (Elaboración del autor, 2016)

\begin{tabular}{|c|c|c|}
\hline MATERIAL & EMISIONES $\left(\mathbf{t C O} \mathbf{~}_{\mathbf{2}}\right)$ & HE (hag) \\
\hline Hormigón & $7.527,876$ & $3.669,578$ \\
\hline PVC & $1.988,227$ & 969,192 \\
\hline Áridos & $1.147,006$ & 559,126 \\
\hline Asfalto & $1.055,005$ & 514,278 \\
\hline Agua & 789,635 & 385,636 \\
\hline Cerámicos & 677,910 & 330,457 \\
\hline Cemento & 567,397 & 276,587 \\
\hline Hierro & 438,220 & 213,617 \\
\hline Acero & 168,235 & 82,011 \\
\hline Bronce & 8,000 & 3,900 \\
\hline Polietileno & 7,021 & 3,423 \\
\hline Pintura & 2,869 & 1,398 \\
\hline Cobre & 1,555 & 0,758 \\
\hline Plastificante & 1,189 & 0,579 \\
\hline Fibra de vidrio & 0,022 & 0,011 \\
\hline Madera & $-3,808$ & $-1,856$ \\
\hline
\end{tabular}

Tabla 5. Resultados por materiales. (Elaboración del autor, 2016)

\section{CASO PRÁCTICO.}

A continuación, se muestra la aplicación de la metodología descrita a un caso práctico, eligiendo para tal fin el Proyecto de Urbanización del Sector Industrial SUB-O-03 UE-1 de Écija (Sevilla, España) (Figura 3), del cual se han obtenido todos los datos técnicos y económicos necesarios.

En este proyecto de urbanización se incluyen los procesos requeridos para llevar a cabo la ejecución de los trabajos de movimiento de tierras, pavimentaciones e instalaciones necesarios para la urbanización de la zona, con el fin de crear un polígono industrial (Figura 4). El presupuesto está constituido por 181 precios básicos, entre los que se encuentran materiales, maquinaria y mano de obra, del que resulta un importe total de $11.557 .028,87 €$, en un ámbito de actuación de $620.256 \mathrm{~m}^{2}$.

Tras la aplicación de toda la metodología a los datos del proyecto, se obtienen los resultados que se resumen en la Tabla 4 y en la Figura 5. En la Tabla 5 se muestran los materiales con mayor repercusión en cuanto a impacto ambiental.

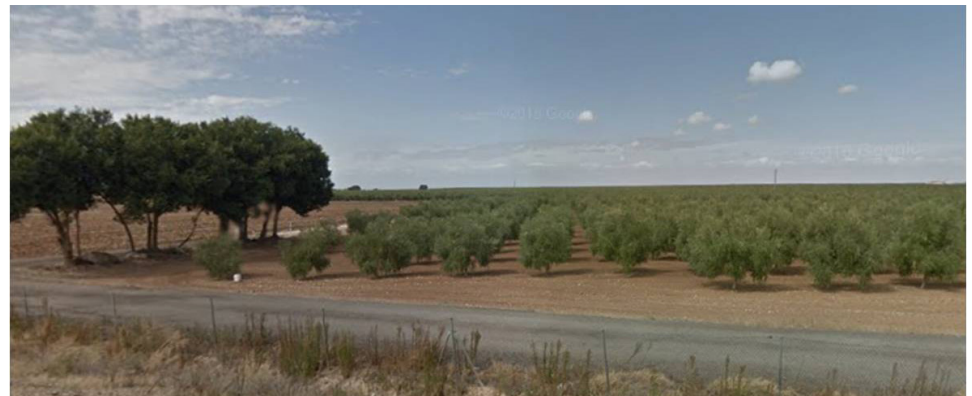

Figura 3. Parcela a urbanizar. (Elaboración del autor, 2016)

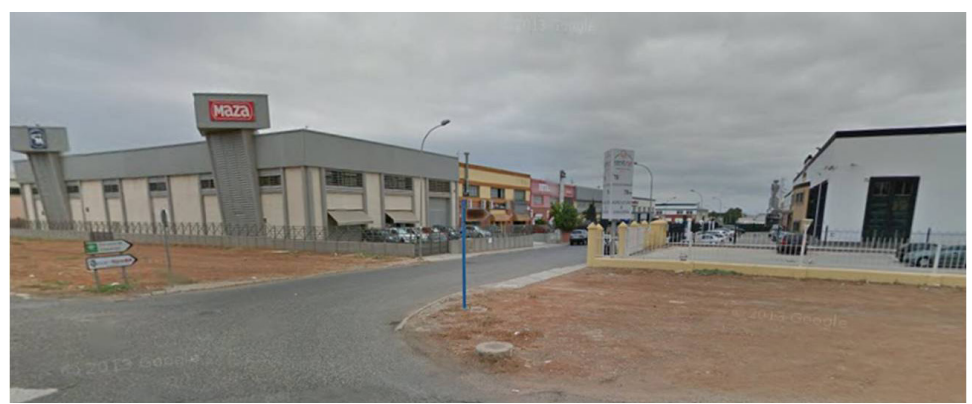

Figura 4. Polígono industrial. (Elaboración del autor, 2016) 


\section{Huella ecológica}

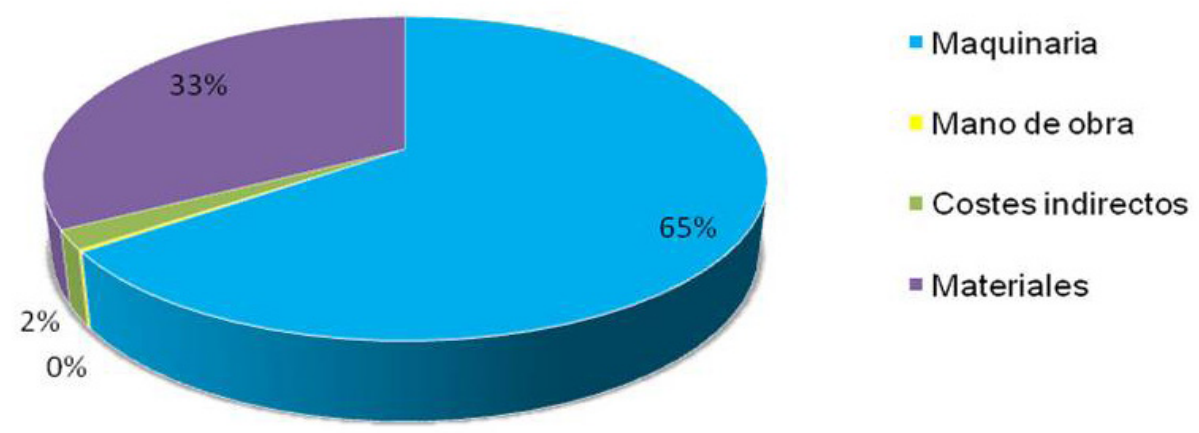

Figura 5. HE, según fuente de impactos. (Elaboración del autor, 2016)

Tras aplicar la metodología al caso práctico, se obtiene una HE total de 19.927,08 hag del proyecto, que al calcularse considerando la superficie de actuación del proyecto deviene en 321,14 hag/ha; huella similar a la de la construcción de viviendas evaluada por otros autores (Solís, 2011; González, Marrero y Solís, 2015). Aunque, a diferencia de ellos, aquí no resultan significativas las huellas relacionadas con la mano de obra y, en concreto, con su alimentación. Se puede apreciar en la Figura 5 que la mayor parte de las huellas viene provocada por el empleo de maquinaria (66\%) y uso de materiales (33\%), correspondiendo primordialmente a HC. La alta repercusión de la maquinaria, en este sentido, se debe a que se trata de un proyecto de urbanización del terreno, que implica la utilización de equipo pesado y una mayor presencia aquellos que se necesitan para la realización de los elementos comunes de las urbanizaciones (asfalto y áridos) y las diferentes instalaciones (pvc y hormigón).

\section{CONCLUSIONES.}

El presente trabajo de investigación demuestra la viabilidad de la incorporación de un indicador ambiental, como es la huella ecológica, dentro de la estructura de costes y presupuestación de los proyectos de obra (tanto de construcción como de urbanización), siguiendo la línea de presupuesto ambiental dentro del mundo de la construcción.

De este modo, se facilita la opción de tomar decisiones en fase de proyecto, que tendrán como resultado la mejora de la "calificación ambiental" de la actividad.

También se comprueba que es posible la incorporación de los indicadores $\mathrm{HC}$ y $\mathrm{HE}$ en el sistema de clasificación sistemática de los bancos de costes de construcción, manteniendo su misma organización. Simplemente, se añaden otros elementos diferenciadores de cada componente del presupuesto (precio básico) que pueda servir para su futura elección a la hora de incorporarlo en el desarrollo de un proyecto, no siempre basada en el aspecto económico.

Queda patente, por último, la cada vez más necesaria incorporación de aspectos ambientales en los trámites y quehaceres diarios de la administración pública, y la fácil adaptación que permite la metodología descrita en este artículo del modelo desarrollado en el sistema de clasificación de la información de construcción ( $\mathrm{SCIC}$ ), que emplea la administración en Andalucía, España. En efecto, el modelo constituye un elemento diferenciador para tomar una serie de decisiones sobre, por ejemplo, criterios de adjudicación de proyectos, aspectos fiscales (rebajas en impuestos de inmuebles, exención de tasas urbanísticas de proyectos), entre otros. 


\section{REFERENCIAS BIBLIOGRÁFICAS.}

BASTIANONI, Simone; GALLI, Alessandro; PULSELLI, Ricardo María y $\mathrm{NICCOLUCCl,} \mathrm{Valentina.} \mathrm{Environmental} \mathrm{and} \mathrm{economic} \mathrm{evaluation} \mathrm{of}$ natural capital appropriation through building construction: practical case study in the Italian context. Ambio, 2007, vol. 36, n' 7 , pp. 559565 .

BIN, Guoshu y PARKER, Paul. Measuring buildings for sustainability: Comparing the initial and retrofit ecological footprint of a century home - The REEP House. Applied Energy, 2012, nº 93, pp. 24-32.

CAMBIO GLOBAL ESPAÑA 2020/50. Sector edificación. Programa Cambio Global España 2020/50 del Centro Complutense de Estudios e Información Medioambiental de la Fundación General de la Universidad Complutense de Madrid, Madrid, 2010.

EUROSTAT. Municipal waste generated by country in selected years (kg per capita), 2015

FREIRE, Antonio y MARRERO, Madelyn. Evaluación a través del presupuesto de la energía incorporada al proyecto de edificación. Hábitat Sustentable, 2015a, vol. 5, n 1, pp. 54-63.

FREIRE, Antonio y MARRERO, Madelyn. Ecological Footprint in Indirect Costs of Construction. Ponencia presentada en II Congreso Internacional y IV Nacional de Construcción Sostenible y Soluciones Ecoeficientes, Escuela Técnica Superior de Arquitectura, Universidad de Sevilla, 2015b.

GONZÁLEZ, Patricia; SOLÍS, Jaime; LLÁCER, Rafael y MARRERO, Madelyn. La construcción de edificios residenciales en España en el período 2007-2010 y su impacto según el indicador Huella Ecológica. Informes de la Construcción, 2015, vol. 67, n539.

GONZÁLEZ, Patricia; MARRERO, Madelyn y SOLÍS, Jaime. The ecological footprint of dwelling construction in Spain. Ecological Indicators, 2015, $\mathrm{n}^{\circ}$ 52, pp. 75-84.

GRUNEWALD, Nicole; GALLI, Alessandro; KATSUNORI, Iha; HALLE, Martine y GRESSOT, Michel. The Ecological Footprint of Mediterranean Diets. International Centre for Advanced Mediterranean Agronomic Studies CIHEAM, 2015, Wach Letter ${ }^{\circ} 32$

INSTITUTO PARA LA DIVERSIFICACIÓN Y AHORRO DE LA ENERGÍA (IDEA). Factores de emisión de CO2, Madrid 2011.

LI, Bing y CHENG, Dong-Jing. Hotel ecological footprint model: Its construction and application. Chinese Journal of Ecology, 2010, $\mathrm{n}^{\circ}$ 29 , pp. 1463-1468.

MARTÍNEZ, Alejandro, SOLÍS GUZMÁN, Jaime, MARRERO Madelyn. LCA databases focused on construction materials: A review. Renewable and Sustainable Energy Reviews, 2016, vol. 58, pp. 565573.

OLGYAY, Víctor. Greenfoot: A tool for estimating the carbon and ecological footprint of buildings. American Solar Energy Society - SOLAR 2008, Including Proc. of 37th ASES Annual Conf., 33rd National Passive Solar Conf., 3rd Renewable Energy Policy and Marketing Conf.: Catch the Clean Energy Wave 8, 2008, pp. 50585062.

DOMÉNECH QUESADA Juan Luis: Huella Ecológica y Desarrollo sostenible. AENOR. Madrid, España. 2007.
SAMADPOUR P, FARYADI Sh. Determination of ecological footprints of dense and high-rise districts, case study of Elahie neighbourhood, Tehran. Journal of Environmental Studies, 2008, vol. 34, $n^{\circ} 45$, pp. 63-72.

SOLÍS, Jaime. Evaluación de la huella ecológica del sector edificación (uso residencial) en la comunidad andaluza. Tesis de Doctorado, Universidad de Sevilla, 2011

SOLÍS, Jaime; MARRERO, Madelyn y RAMÍREZ DE ARELLANO, Antonio. Methodology for determining the ecological footprint of the construction of residential buildings in Andalusia (Spain). Ecological Indicators, 2013, $\mathrm{n}^{\circ} 25$, pp.239-249.

SOLÍS, Jaime; MARTÍNEZ, Alejandro y MARRERO, Madelyn. Methodology for determining the carbon footprint of the construction of residential buildings. Assessment of Carbon Footprint in Different Industrial Sectors, Volume 1. Springer, 2014, pp. 49-83.

SOLÍS, Jaime; GONZÁLEZ, Patricia; MARTíNEZ, Alejandro; MARRERO, Madelyn. The Carbon Footprint of Dwelling Construction in Spain. The Carbon Footprint Handbook. CRC Press - Taylor \& Francis Group, 2015, pp. 261-283.

TENG, Jiaying y WU, Xianguo. Eco-footprint-based life-cycle ecoefficiency assessment of building projects. Ecological Indicators, 2014, n³9, pp. 160-168.

WACKERNAGEL, Mathis y REES, William. Our Ecological Footprint: Reducing Human Impact on the Earth. Canadá: New Society Publishers, 1996.

ZHAO, Xueyan y MAO, Xiaowen. Comparison environmental impact of the peasant household in han, zang and hui nationality region: Case of zhangye, Gannan and Linxia in Gansu Province. Shengtai Xuebao/Acta Ecologica Sinica, 2013, vol. 33, n 17, pp. 5397-5406. 